\title{
BeppoSAX observation of the eclipsing dipping $X$-ray binary X 1658-298
}

\author{
T. Oosterbroek ${ }^{1}$, A. N. Parmar ${ }^{1}$, L. Sidoli ${ }^{1}$, J. J. M. in 't Zand ${ }^{2,3}$, and J. Heise ${ }^{3}$ \\ 1 Astrophysics Division, Space Science Department of ESA, ESTEC, PO Box 299, 2200 AG Noordwijk, \\ The Netherlands \\ 2 Astronomical Institute, Utrecht University, PO Box 80 000, 3508 TA Utrecht, The Netherlands \\ 3 Space Research Organization Netherlands, Sorbonnelaan 2, 3584 CA Utrecht, The Netherlands
}

Received 8 January 2001 / Accepted 5 July 2001

\begin{abstract}
Results of a 2000 August 12-13 BeppoSAX observation of the $7.1 \mathrm{hr}$ eclipsing, dipping, bursting, transient, low-mass X-ray binary (LMXRB) X 1658-298 are presented. The spectrum outside of eclipses, dips and bursts can be modeled by the combination of a soft disk-blackbody and a harder Comptonized component with a small amount $\left(1.3 \times 10^{21}\right.$ atom $\left.\mathrm{cm}^{-2}\right)$ of low-energy absorption. In contrast, an RXTE observation 18 months earlier during the same outburst, measured an absorption of $5.0 \times 10^{22}$ atom $\mathrm{cm}^{-2}$. Such a change is consistent with a thinning of the accretion disk as the outburst progresses. Structured residuals from the best-fit spectral model are present which are tentatively identified with Ne-K/Fe-L and Fe-K shell emission. The spectral changes during dips are complex and may be modeled by a strong $\left(\sim 3 \times 10^{23}\right.$ atom $\left.\mathrm{cm}^{-2}\right)$ increase in absorption of the Comptonized component only, together with reductions in normalizations of both spectral components. This behavior is in contrast to the "complex continuum" model for X-ray dip sources, where the softer blackbody component rapidly suffers strong absorption. It is however, similar to that found during recent XMM-Newton observations of the eclipsing, dipping, LMXRB EXO 0748-676. An updated orbital ephemeris is provided which does not require a quadratic term, in contrast to that of Wachter et al. (2000).
\end{abstract}

Key words. accretion, accretion disks - stars: individual: X 1658-298 - stars: neutron - X-rays: stars

\section{Introduction}

$\mathrm{X} 1658-298$ is a transient X-ray source discovered by SAS-3 (Lewin et al. 1976). Bursts were reported from X 1658-298 in 1976 October and 1977 June, but there was apparently no significant persistent emission (Lewin 1979). During 1978 March, observations with SAS-3 and HEAO-1 detected a clearly modulated persistent emission which showed erratic intensity variations followed by 15 minute duration X-ray eclipses which repeated periodically (Cominsky \& Wood 1984). By combining data spanning $\sim 2$ years an orbital period of $7.11 \mathrm{~h}$ was obtained (Cominsky \& Wood 1989). Following the discovery using EXOSAT of a second bursting, dipping, eclipsing, transient (EXO 0748-676; Parmar et al. 1986) the region of sky containing X 1658-298 was observed by EXOSAT. However, the source was not detected. Indeed, until recently X 1658-298 has not been observed to be active. In 1991 a 25 ks ROSAT Position Sensitive Proportional

Send offprint requests to: T. Oosterbroek, e-mail: toosterb@astro.estec.esa.nl
Counter observation failed to detect X 1658-298 with a $0.2-2.4 \mathrm{keV}$ upper limit of $\sim 2 \times 10^{-14} \mathrm{erg} \mathrm{cm}^{-2} \mathrm{~s}^{-1}$. However, on 1999 April 2, after a 21 year quiescent interval, an 18 mCrab (2-9 keV) source at a position consistent with X 1658-298 was detected in one of the BeppoSAX Wide Field Cameras (in 't Zand et al. 1999a). Observations a day later confirmed that the $V=18.3 \mathrm{op}-$ tical counterpart to X 1658-298 (V2134 Oph; Doxsey et al. 1979) had brightened significantly and was exhibiting high excitation He II emission, confirming that the source was undergoing an outburst (Augusteijn et al. 1999).

X 1658-298 was observed by RXTE a few days later (Wachter et al. 2000, hereafter WSB) confirming that the source had resumed its strong and persistent X-ray emission. WSB combined their RXTE eclipse timing measurements with those obtained with HEAO A-1 and SAS-3 and found that the orbital period of X 1658-298 had decreased with a timescale of $10^{7} \mathrm{yr}$. The eclipse transitions of 6 to $13 \mathrm{~s}$ were clearly resolved in the RXTE measurements. Contemporaneous observations revealed that the optical modulation is highly variable, and exhibits a 


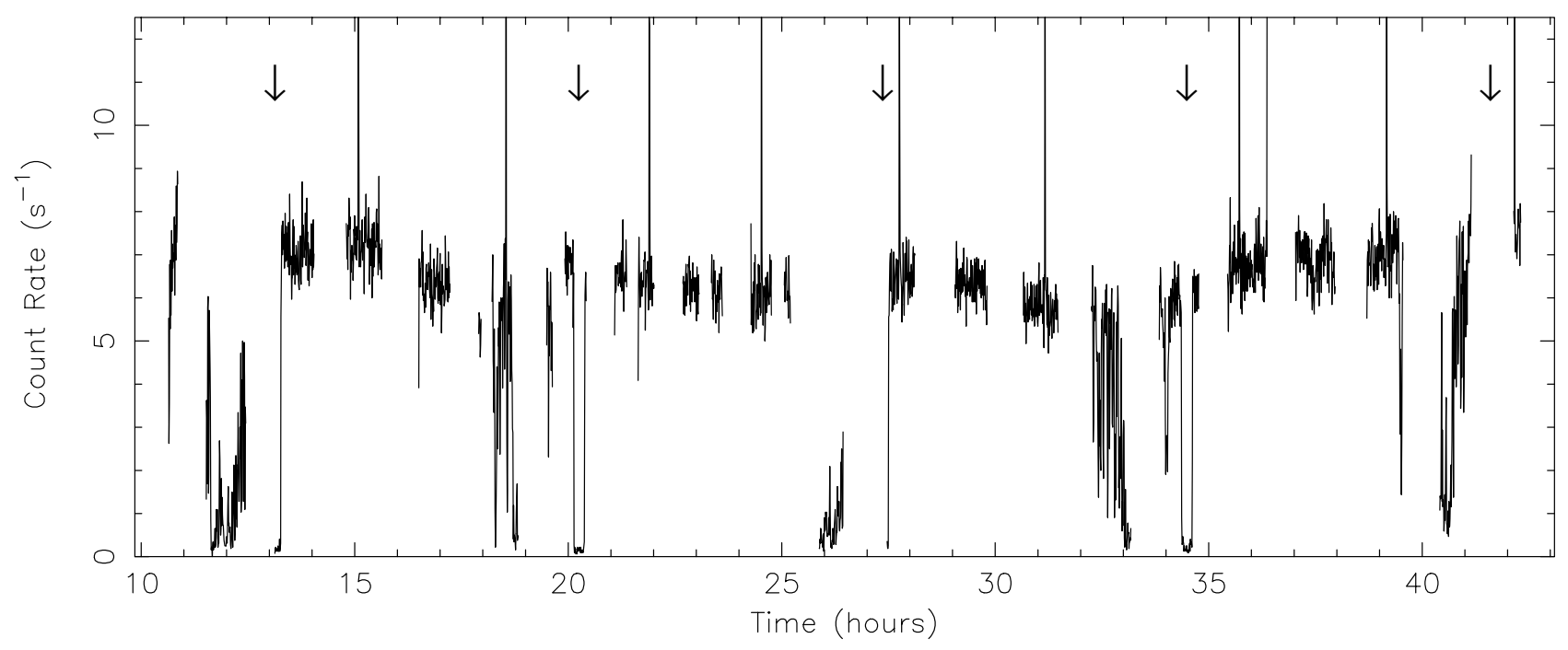

Fig. 1. 2-10 keV MECS lightcurve of X 1658-298 with a binning of 32 s. 2 completely and 2 partially observed eclipses, irregular intensity dips and 9 X-ray bursts are evident. The peak heights of the bursts are truncated. The arrows mark the expected mid-eclipse times. Time is hours of 2000 August 12.

0.2 mag eclipse feature coincident with the X-ray eclipse. Recently, nearly coherent pulsations were discovered from this source during Type I X-ray bursts (Wijnands et al. $2000)$. These imply a spin period of $\sim 1.8 \mathrm{~ms}$, if the oscillations are directly related to the neutron star rotation.

Only two Low-Mass X-ray Binary (LMXRB) systems are known that exhibit both dips and eclipses. (About 10 LMXRBs exhibit only dips.) During dips the observed spectral changes are complex and inconsistent with simple absorption from cold material, as might be expected. Detailed modeling of these spectral changes provides a powerful means of studying the structure and location of the emitting and absorbing regions in LMXRB (e.g., Parmar et al. 1986; Church \& Bałucińska-Church 1995; Church et al. 1997). This modeling has revealed that at least two emission components are required. These regions appear to undergo different amounts of absorption during dips with a point-like blackbody undergoing rapid obscuration and a cutoff power-law component undergoing a more gradual increase in obscuration during dipping intervals. This gradual change is highly suggestive of an extended component. We refer to this as the complexcontinuum model. The same spectral model has been used to describe the ASCA spectra of a large number of LMXRBs (Church \& Bałucińska-Church 2001).

In the case of EXO0748-676, (the other eclipsing and dipping LMXRB), recent XMM-Newton Reflection Grating Spectrometer (RGS) observations have revealed the presence of narrow spectral features due to ionized neon, oxygen and nitrogen (Cottam et al. 2001). Simultaneous European Photon Imaging Camera (EPIC) data revealed that the $0.2-10 \mathrm{keV}$ spectra can be fit with a two component model consisting of a central Comptonized component and a more extended thermal halo (BonnetBidaud et al. 2001). In contrast to the complex continuum continuum model, the spectral variations observed during dips can be mainly accounted for by variations in the absorbing column affecting the Comptonized component only.

\section{Observations}

Results from the Low-Energy Concentrator Spectrometer (LECS; 0.1-10 keV; Parmar et al. 1997), the MediumEnergy Concentrator Spectrometer (MECS; 1.8-10 keV; Boella et al. 1997), the High Pressure Gas Scintillation Proportional Counter (HPGSPC; 5-120 keV; Manzo et al. 1997) and the Phoswich Detection System (PDS; 15-300 keV; Frontera et al. 1997) on-board BeppoSAX are presented. All these instruments are coaligned and collectively referred to as the Narrow Field Instruments, or NFI. The MECS consists of two grazing incidence telescopes with imaging gas scintillation proportional counters in their focal planes. The LECS uses an identical concentrator system as the MECS, but utilizes an ultra-thin entrance window to extend the response down to $0.1 \mathrm{keV}$. The non-imaging HPGSPC consists of a single unit with a collimator that was pointed on-source during the whole observation. The non-imaging PDS consists of four independent units arranged in pairs each having a separate collimator. Each collimator was alternatively rocked onand $210^{\prime}$ off-source every $96 \mathrm{~s}$ during the observation.

The region of sky containing X 1658-298 was observed by BeppoSAX on 2000 August 12 10:33 UT to August 13 18:14 UT. Good data were selected in the standard way using the SAXDAS 2.0.0 data analysis package. LECS and MECS data were extracted centered on the (on-axis) position of X 1658-298 using radii of $8^{\prime}$ and $4^{\prime}$, respectively. The exposure times in the LECS, MECS, HPGSPC, and PDS instruments are $20.2 \mathrm{ks}, 53.5 \mathrm{ks}, 50.1 \mathrm{ks}$, and $24.3 \mathrm{ks}$, respectively. Background subtraction for the imaging instruments was performed using standard files, but is not critical for such a bright source. Background subtraction 


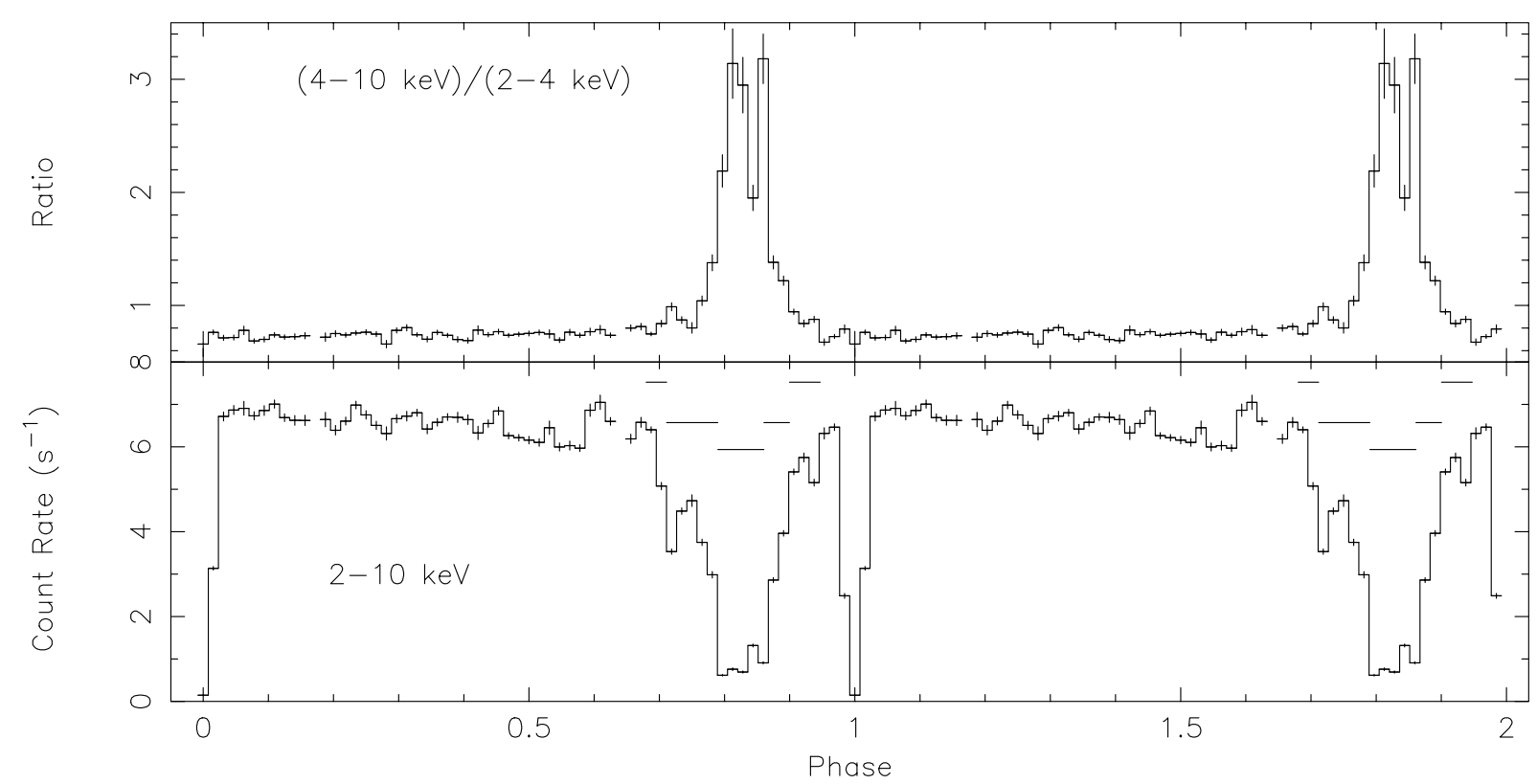

Fig. 2. The folded hardness ratio (top panel) and 2-10 keV MECS lightcurve showing the deep dipping activity between phases $\sim 0.68-0.95$ and the narrow eclipse centered on phase 0 . X-ray bursts have been removed prior to the folding. The horizontal lines indicate phases used to extract the three dip-spectra (see Sect. 5.2). The central line corresponds to the deep dip interval, the two middle lines to the medium dipping, and the two outer lines to the shallow dipping interval.

for the HPGSPC was carried out using data obtained when the instrument was observing the dark Earth and for the PDS using data obtained when the collimator was offset from the source.

\section{X-ray lightcurve}

Figure 1 shows the $2-10 \mathrm{keV}$ MECS lightcurve of X 1658298 with a binning of $32 \mathrm{~s}$ spanning just over 4 orbital cycles. As expected, eclipses, irregular dips in X-ray intensity, and bursts are present. Neither the dips nor the eclipses are quite total. A folded $2-10 \mathrm{keV}$ lightcurve with bursts removed, and the (4.0-10.0 keV/2.0-4.0 keV) hardness ratio as a function of phase are plotted in Fig. 2. From this figure it is evident that strong dipping behavior occurs between phases $0.68-0.95$ (where center of $\mathrm{X}$-ray eclipse corresponds to phase 0.0). At other phases, the hardness ratio increases slowly with increasing phase, from a minimum around phase 0.0. This may indicate that low-level dipping is present throughout the orbital cycle. Alternatively, this variation could be due to a dependence of the X-ray emission on orbital phase.

\section{Eclipse timing}

The arrival times of the eclipses were determined by fitting a "step and ramp" function (see Parmar et al. 1986) to the MECS light curves (which were obtained at $8 \mathrm{~s}$ resolution) of the two complete eclipses. This model has 7 free parameters: the start- and end-times of the ingress and egress, and the count rates before, during, and after eclipse. The time of eclipse was calculated as the mean between the end time of ingress and the start time of egress.
The derived eclipse times, which extend the measurements of WSB by more than a year, or 1600 cycles, are summarized in Table 1. These times were then combined with the eclipse times compiled by WSB and linear and quadratic ephemerides investigated. Figure 3 shows the residuals with respect to both ephemerides. Since the WSB results require a quadratic solution (see below) a quadratic ephemeris was first fit to the eclipse times to obtain a $\chi^{2}$ of 45.4 for 5 degrees of freedom (d.o.f.). The updated quadratic ephemeris for the eclipse centers is given by:

$$
\begin{aligned}
T_{\text {ecl }}= & 2443059.22609(11)+0.29650453(33) N \\
& +(1.1 \pm 0.3) \times 10^{-12} N^{2} .
\end{aligned}
$$

Note that the sign of the quadratic term is opposite from that of WSB, and the magnitude a factor $\sim 10$ smaller. A linear ephemeris was next fit to the eclipse times to give a $\chi^{2}$ of 62.9 for 6 d.o.f.:

$T_{\text {ecl }}=2443059.225826(93)+0.2965045746(34) N$.

The $\chi^{2}$ values of the two fits indicate that the difference in $\chi^{2}$ is not significant (the probability of finding a decrease in the $\chi^{2}$ by this value (or larger) is $22 \%$ ). Note that the $\chi_{\nu}^{2}$ values are much larger than 1 , indicating that neither fit is formally acceptable.

To investigate the large difference in the quadratic terms of the two ephemerides, only the data points reported in WSB were fit. This gives the same solution as WSB found (Fig. 4). The WSB solution goes through the two sets of data points, but deviates strongly from the linear ephemeris in the interval where no measurements are available. From this we conclude that the ephemeris obtained by WSB is an artifact of the strongly non-uniform 


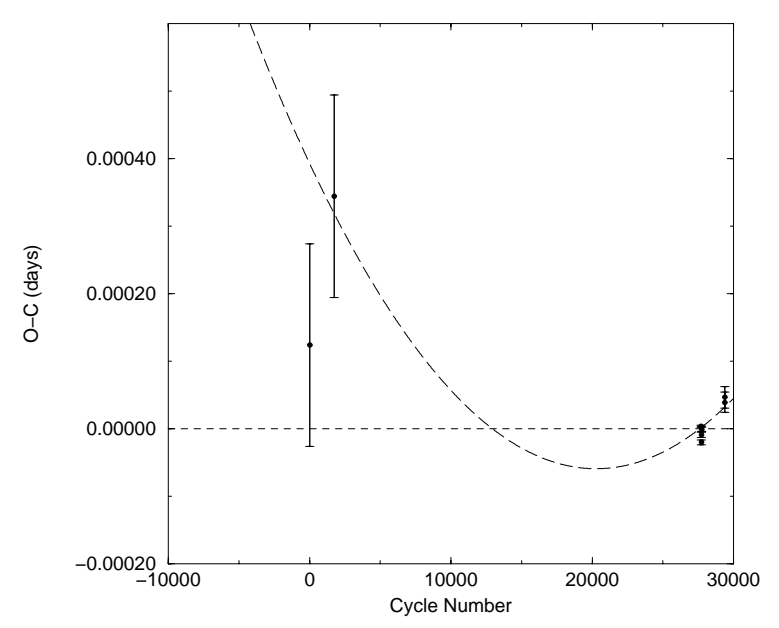

Fig. 3. The X 1658-298 eclipse timing residuals with respect to the best-fit linear ephemeris. The BeppoSAX measurements are the two most recent data points. The best-fit quadratic ephemeris (long dashed line) is also indicated.

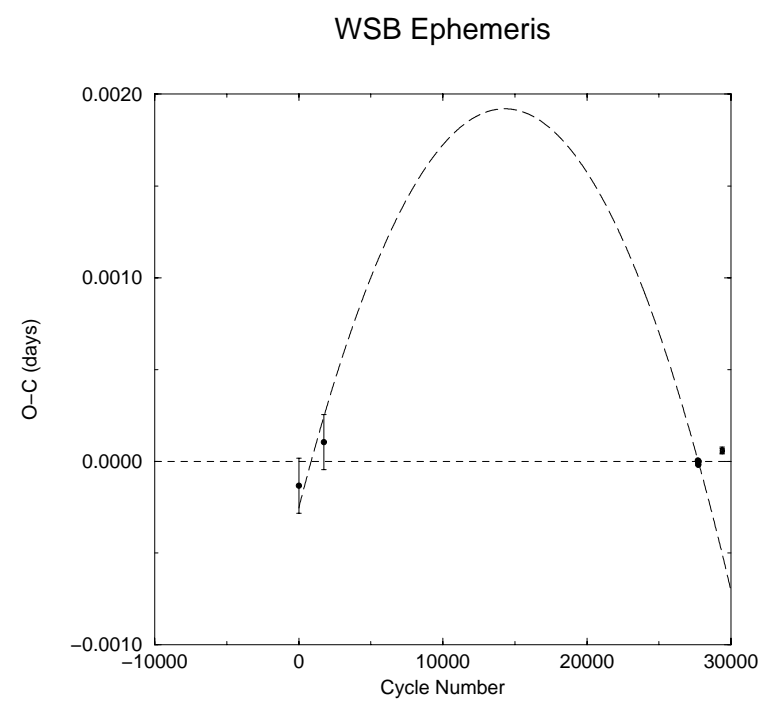

Fig. 4. The X 1658-298 eclipse timing residuals with respect to a linear ephemeris obtained using the WSB data only. The WSB quadratic ephemeris is indicated by the long-dashed line. The BeppoSAX data points are strongly inconsistent with the WSB quadratic ephemeris. Note the different vertical scale with respect to Fig. 3 .

sampling of the data and that their $\dot{P}$ has no physical relevance. In Fig. 3 the linear ephemeris when the BeppoSAX data are included is plotted. From this we conclude that a linear ephemeris is an acceptable solution, although deviations (of the order of a few $\sigma$ ) are present. These may be similar to the "random" deviations in eclipse times seen from EXO 0748-676 (Hertz et al. 1997).

\section{X-ray spectrum}

\subsection{Overall spectrum}

The overall spectrum of X1658-298 was first investigated by simultaneously fitting data from all the BeppoSAX NFI. Only intervals when the source was not
Table 1. New X-ray center of eclipse times (corrected to the solar system barycenter) for X 1658-298. A compilation of previous measurements can be found in WSB. Cycle numbers are given using the convention in WSB. Uncertainties are given at $68 \%$ confidence.

\begin{tabular}{lll}
\hline $\mathrm{JD}_{\odot}$ & Uncertainty & Cycle \\
\hline 2451769.344257 & 0.000016 & 29376 \\
2451769.937259 & 0.000015 & 29378 \\
\hline
\end{tabular}

bursting, dipping, or eclipsing were included. This resulted in exposure times in the LECS, MECS, HPGSPC, and PDS instruments of $13.0,30.8,27.6$, and $13.5 \mathrm{ks}$, respectively. In order to ensure applicability of the $\chi^{2}$ statistic, the extracted spectra were rebinned such that at least 20 counts per bin were present and such that the resolution of the instrument is not oversampled by more than a factor 3. LECS data were used in the energy range 0.5-4.0 keV, MECS data between 1.85-10.5 keV, HPGSPC data between 8-20 keV, and PDS data in the 15-30 keV energy range, where the instrument responses are well determined and sufficient counts obtained. This gives background-subtracted count rates of 3.5, 6.8, 3.1 and $0.7 \mathrm{~s}^{-1}$ in these energy ranges for the LECS, MECS, HPGSPC, and PDS, respectively. The photo-electric absorption cross sections of Morrison \& McCammon (1983) were used throughout. Factors were included in the spectral fitting to allow for normalization uncertainties between the instruments. These factors were constrained to be within their usual ranges during the fitting. All spectral uncertainties and upper-limits are given at $90 \%$ confidence. A systematic uncertainty of $1 \%$ was added quadratically to account for known instrument calibration uncertainties.

Since the source is $7^{\circ}$ away from the galactic plane we verified that emission from the galactic ridge (e.g., Valinia \& Marshall 1998) is unlikely to affect the background determination in the non-imaging instruments. The HPGSPC background data are obtained during observations of the dark Earth and therefore the resultant spectrum will be the sum of the source spectrum and the galactic ridge spectrum. However, given the large offset from the galactic plane and the brightness of the source, this is unlikely to be a significant effect. The PDS background spectra are obtained by rocking the instrument collimator 3.5 in opposite directions. Thus, changes in the galactic ridge emission (as a function of galactic latitude) could lead to an imperfect background subtraction. We therefore accumulated PDS spectra using only the positive and negative offset background directions. The differences in the PDS spectra obtained in this way are much smaller than the statistical uncertainties.

The resulting spectra were first fit with simple models including absorbed power-laws with photonindex $\alpha$, thermal bremsstrahlung and cutoff power-laws $\left(E^{-\alpha} \exp -\left(E_{\mathrm{c}} / k T\right)\right)$. This showed that no such model 

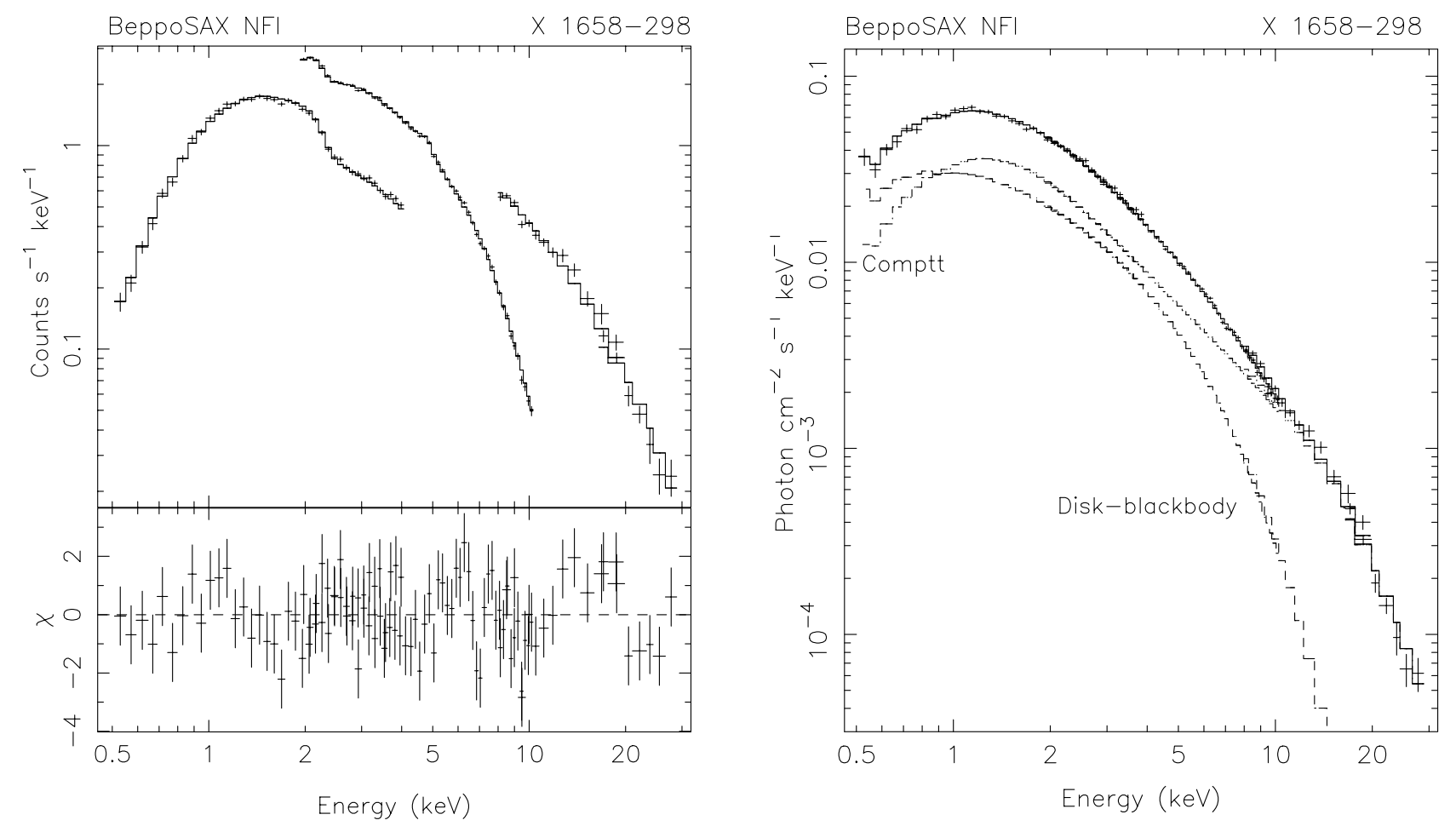

Fig. 5. The 0.5-30 keV non-dip, non-burst, and non-eclipse ("persistent emission") X 1658-298 count spectrum and residuals when a disk-blackbody and COMPTT model are fit. The right hand panel shows the deconvolved photon spectrum with the contributions of the two components indicated.

provides an acceptable fit with e.g., an absorbed powerlaw with $\alpha \sim 2$ giving a $\chi^{2}$ of 1149 for 103 d.o.f. A cutoff power-law with $\alpha=1.3$ and absorption, $N_{\mathrm{H}}$, of $3.5 \times 10^{21}$ atom $\mathrm{cm}^{-2}$ provides a significantly better fit with a $\chi^{2}$ of 231 for 102 d.o.f. implying the presence of a high-energy break at $6.1 \mathrm{keV}$. More complex models, consisting of combinations of soft and hard components were next tried. The soft components tried were blackbodies, collisionally ionized thermal plasmas (the MEKAL model in XSPEC), and the disk-blackbody model of Mitsuda et al. (1984). This model assumes that the gravitational energy released by the accreting material is locally dissipated into blackbody radiation, that the accretion flow is continuous throughout the disk, and that the effects of electron scattering are negligible. There are only two parameters of the model, $r_{\mathrm{in}}(\cos i)^{0.5}$ where $r_{\mathrm{in}}$ is the innermost radius of the disk, $i$ is the inclination angle of the disk and $k T_{\text {in }}$ the blackbody effective temperature at $r_{\text {in }}$. The hard components tried were power-laws, cutoff power-laws and the XSPEC COMPTT Comptonization model described in Titarchuk (1994). This model contains as free parameters the temperature of the Comptonizing electrons $k T_{\mathrm{e}}$, the plasma optical depth with respect to electron scattering $\tau_{\mathrm{e}}$ and the input temperature of the soft photon (Wien) distribution $k T_{\mathrm{W}}$. A spherical geometry was assumed for the comptonizing region. Of all the possible combinations, the best-fit two component model is a diskblackbody and a COMPTT component with a $\chi^{2}$ of 138.6 for 99 d.o.f. (Fig. 5). The absorption corrected $2-10 \mathrm{keV}$ luminosity is $1.6 \times 10^{37} \mathrm{erg} \mathrm{s}^{-1}$ for a distance of $15 \mathrm{kpc}$
(Cominsky 1981). Table 2 gives the fit results for models with $\chi_{\nu}^{2}<2$. The absorption derived using the bestfit model of $(1.3 \pm 0.2) \times 10^{21}$ atom $\mathrm{cm}^{-2}$ is similar to the galactic absorption in the direction of X 1658-298 of $1.8 \times 10^{21}$ atom $\mathrm{cm}^{-2}$ (Dickey \& Lockman 1990).

We note here that we only use the COMPTT model as a convenient fit model. The physical interpretation of the obtained parameters (which we do not attempt to do) is difficult. The COMPTT model assumes the Wienapproximation for the input photons; for large values of $k T_{0}$ and low energies this breaks down. In our case the COMPTT model underestimates the flux up to $40 \%$ at the lowest energies (see Church \& Bałucińska-Church 2001). Additionally, there is evidence that the size of the Comptonized region is quite large (typically $50000 \mathrm{~km}$; Church 2001). The temperature of the seed-photons if they originate from the disk at these distances is much lower than best-fit seed-temperature for typical luminosities. Additionally, the difference in shape (at low energies) as discussed above will influence the normalization and parameters of the second (disk-blackbody) component, making a physical interpretation of these parameters even harder.

Interestingly, recent results from the XMM-Newton EPIC show that the soft component seen from EXO 0748676 may be modeled by a collisionally ionized thermal plasma with strongly non-solar abundances (BonnetBidaud et al. 2001). This is not the case in X 1658298 , where replacing the disk-blackbody by a $\sim 1.4 \mathrm{keV}$ MEKAL component with abundances fixed at solar gives 
a significantly worse fit with a $\chi^{2}$ of 241.8 for 99 d.o.f. Allowing the abundances to vary does not provide a significantly improved fit. Additionally, Bonnet-Bidaud et al. (2001) find that their EXO 0748-676 power-law component suffers extra absorption of $\sim 6 \times 10^{22}$ atom $\mathrm{cm}^{-2}$ during non-dipping or flaring intervals. No such additional absorption, with a $90 \%$ confidence upper limit of $9 \times 10^{20}$ atom $\mathrm{cm}^{-2}$, is required by the $\mathrm{X} 1658-298$ COMPTT component during non-dipping intervals.

We next investigated the reasons for the poor $\left(\chi^{2}=\right.$ 138.6 for 99 d.o.f.) best-fit. Inspection of Fig. 5 reveals the presence of structured residuals, with amplitudes of $\sim 5 \%$ with similar forms for the different instruments in the overlapping energy ranges. This indicates that the observed inadequacies of the spectral models may be physical, and not caused by incorrectly modeled instrumental responses. In order to investigate the nature of the residuals we selected spectra (i) based on count rate, and (ii) based on orbital phase. The first check was performed in order to see if the residuals are somehow caused by accumulating data from a range of source intensities. However, no difference in the structure of the residuals was found between the high and low count rate selected spectra. The second test was performed since it appears that the hardness ratio increases close to the deep dipping phases (see Fig. 2). Again, no difference in the structure of the residuals was found between spectra obtained at "early" and "late" phases. We therefore conclude that the observed residual structure is probably an intrinsic feature of the X 1658-298 spectrum.

Finally, we tried the ad-hoc addition of Gaussian emission features. Including narrow features at $1.06 \pm 0.08$ and $6.23 \pm 0.13 \mathrm{keV}$ with equivalent widths of $26 \pm 16$ and $41 \pm{ }_{9}^{17} \mathrm{eV}$ reduced the $\chi^{2}$ to 114.7 for 95 d.o.f. The probability of obtaining a decrease in $\chi^{2}$ by this value (or larger) is $1.2 \%$. These features may be tentatively identified with $\mathrm{Ne}-\mathrm{K} / \mathrm{Fe}-\mathrm{L}$ and Fe-K shell emission. Inspection of the remaining residuals suggests that other discrete spectral features may be present in the spectrum. Given that XMM-Newton RGS observations have revealed $\mathrm{O}$, Ne, and $\mathrm{N}$ features in the spectrum of the other eclipsing, dipping, LMXRB EXO 0748-676 (Cottam et al. 2001), this is perhaps unsurprising. We verified that adding similar Gaussians features to the other 3 models listed in Table 2 does not produce markedly better fits. In all cases the change in $\chi^{2}$ when the Gaussians were included was similar to that of the best-fit model.

\subsection{Dip spectrum}

In order to understand the spectrum of a dip source, a suitable model must give acceptable fits to both non-dip and dipping intervals. Since dips are almost certainly due to obscuration by intervening material, such a model should fit the dip spectra without requiring any of the parameters that characterize the shape of the emission, such as the temperature or power-law slope to be changed. These
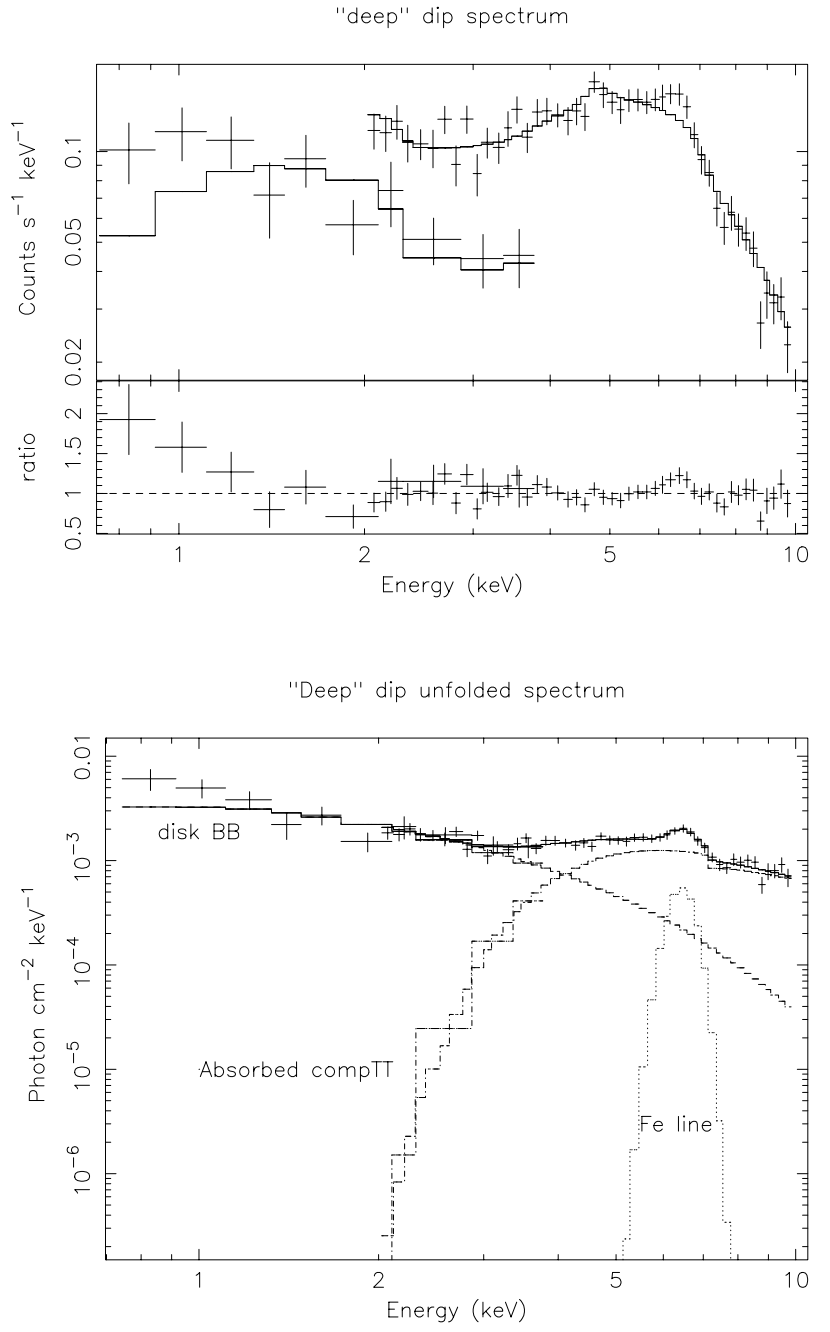

Fig. 6. Top panel: The LECS and MECS spectra of X 1658-298 obtained during the deep dip (see text). The fit consists of the model described in the text, without the addition of the Fe-K line to clearly show the residuals near $6.4 \mathrm{keV}$. Lower panel: the photon spectrum during the deep dip with the separate components indicated. Compare this with Fig. 5 to see the large effect of absorption on the COMPTT component. The Fe line is included in the model for this panel.

parameters are usually obtained by fitting to the non-dip spectrum. In the case of the "complex-continuum" spectral models, which have been used to model the spectral changes seen in many of the dip sources, the emission consists of a point-like blackbody plus an extended Comptonized component. During dips the blackbody is rapidly absorbed, consistent with it being point-like, while the Comptonized component is more progressively absorbed, consistent with it being extended (e.g., Church \& Bałucińska-Church 1995; Church et al. 1997). The blackbody is usually assumed to originate from the surface of the neutron star or from a boundary layer between the neutron star and an accretion disk, and the extended component from an isothermal accretion disk corona.

In order to investigate the spectral changes during X 1658-298 dipping intervals, three sets of LECS and 


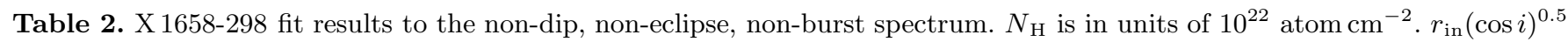
is in units of $\mathrm{km}$ for a distance of $15 \mathrm{kpc}$. $90 \%$ confidence limits are given. $\mathrm{CPL}=$ cutoff power-law, CTT $=\mathrm{COMPTT}, \mathrm{BB}=$ blackbody, DBB = disk-blackbody. All temperatures are in keV. The normalization ("XSPEC units") of the COMPTT component in the best-fit $(\mathrm{DBB}+\mathrm{CTT})$ model is $(3.51 \pm \underset{0.48}{0.30}) \times 10^{-2}$.

\begin{tabular}{lccccccccc}
\hline Model & $N_{\mathrm{H}}$ & $k T_{\mathrm{BB}} / k T_{\mathrm{in}}$ & $r_{\mathrm{in}}(\cos i)^{0.5}$ & $\alpha$ & $E_{\mathrm{c}}(\mathrm{keV})$ & $k T_{\mathrm{w}}$ & $k T_{\mathrm{e}}$ & $\tau_{\mathrm{e}}$ & $\chi^{2} /$ d.o.f. \\
\hline BB+CPL & $0.29 \pm 0.03$ & $0.77 \pm 0.07$ & $\ldots$ & $1.25 \pm 0.07$ & $7.1 \pm 0.9$ & $\ldots$ & $\ldots$ & $\ldots$ & $167.1 / 100$ \\
DBB+CPL & $0.27 \pm 0.03$ & $1.17 \pm 0.15$ & $3.7 \pm 0.8$ & $1.09 \pm 0.16$ & $7.2 \pm 1.6$ & $\ldots$ & $\ldots$ & $\ldots$ & $158.7 / 100$ \\
BB+CTT & $0.07 \pm 0.02$ & $0.49 \pm 0.01$ & $\ldots$ & $\ldots$ & $\ldots$ & $0.92 \pm_{0.11}^{0.05}$ & $4.8 \pm 0.9$ & $3.5 \pm 0.6$ & $144.5 / 99$ \\
DBB+CTT & $0.13 \pm 0.02$ & $1.63 \pm 0.13$ & $2.3 \pm{ }_{0.4}^{0.5}$ & $\ldots$ & $\ldots$ & $0.39 \pm 0.03$ & $4.0 \pm 0.3$ & $5.4 \pm 0.6$ & $138.6 / 99$ \\
\hline
\end{tabular}

MECS spectra were generated corresponding to shallow, medium, and deep dipping intervals (see Fig. 6 for a spectrum during the deep dip). Selection was made using the orbital phases indicated in Fig. 2. The MECS 2-10 keV count rates were $5.4,3.4$, and $0.8 \mathrm{~s}^{-1}$ with exposures of $5.2,5.4$, and $4.2 \mathrm{ks}$, respectively. Care was taken to minimize the range of count rates included in each accumulation, since this could lead to "mixing" of intervals with different spectra. Due to the low count rates and exposure times HPGSPC and PDS data were not included.

\subsubsection{Simple absorption}

Initially, the best-fit persistent emission model, modified to allow additional absorption to both components, was fit to the dip spectra. It was not possible to obtain satisfactory fits. Inspection of the residuals revealed that significant soft excesses were present in all 3 dip spectra. These can best be modeled if the absorptions $\left(N_{\mathrm{H}}^{\mathrm{CTT}}\right.$ and $\left.N_{\mathrm{H}}^{\mathrm{DBB}}\right)$ and normalizations $\left(I_{\mathrm{CTT}}\right.$ and $\left.I_{\mathrm{DBB}}\right)$ of the diskblackbody and COMPTT components are allowed to vary separately. This is the simplest model that is able to adequately model the spectral changes during dipping intervals and is referred to here as the "simple" absorption model.

The results of fitting this simple absorption model are reported in Table 3 and show that during dips the normalizations of both components decrease, while only the COMPTT component is affected by strong additional absorption. The depth of dipping is determined primarily by the reductions in normalization and not by an increase in absorption. In Fig. 7 the normalizations of both components are plotted as a function of the total flux in the dip spectra. This shows that the disk-blackbody normalization has a stronger dependence on total flux than the COMPTT component decreasing to a value of $\sim 5 \%$ of the persistent emission during the deep dip. In contrast, the COMPTT component has a value $\sim 60 \%$ of the persistent normalization during the deep dip.

We investigated the sudden "onset" of the absorption (i.e. even the shallow-dip spectrum shows COMPTT absorption of $3.4 \times 10^{23}$ atom $\mathrm{cm}^{-2}$ ) by fitting the quiescent spectrum with the same simple absorption model. We were unable to obtain a fit to this spectrum with strong extra absorption for either component, but instead the best-fit

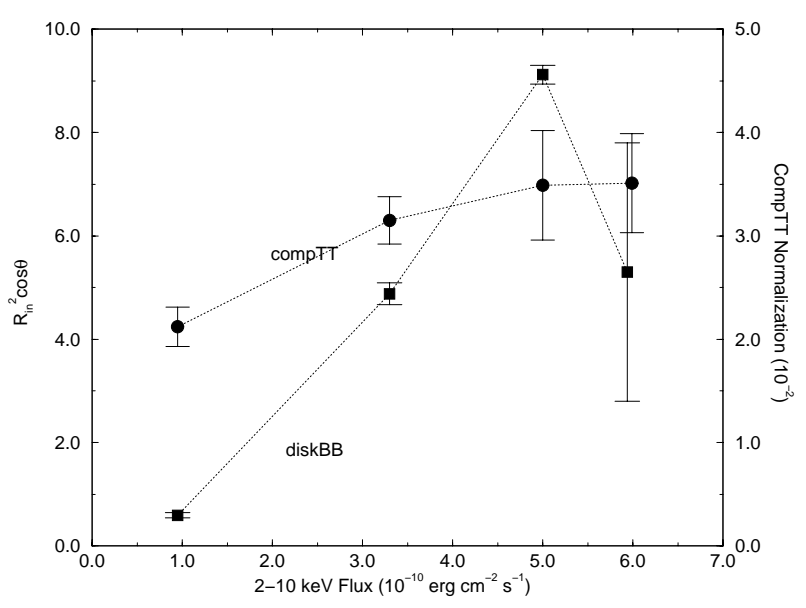

Fig. 7. The normalizations of both the disk-blackbody $\left(r_{\text {in }}^{2} \cos i\right.$, left vertical scale, filled squares) and cOMPTT components (right vertical scale, filled circles). From left to right the points correspond to the deep dip, medium dip, shallow dip, and persistent spectra. The rightmost circle has been shifted by a small amount for clarity. See text for a discussion on the value and uncertainty for the rightmost (i.e. persistent) disk-blackbody normalization. The points are connected with dotted lines to guide the eye.

was identical to that reported in Table 2. We also experimented with several other selections to obtain the dip spectra (using only 2 phase intervals, or using 4 intervals within the dip based on count rate). All these selections gave similar results to those described above.

We also tried fitting the dip spectra with the same model, but with either the temperature of the COMPTT component $\left(k T_{\mathrm{e}}\right)$ or the disk-blackbody temperature $\left(k T_{\text {in }}\right)$ as free parameters. This gives almost identical results to those given in Table 3, with the possible exception of the fit to the deep-dip spectrum when $k T_{\mathrm{e}}$ is treated as a free parameter. This may be applicable if e.g., different parts of a structured emitting region are covered during dipping. This gives a $\chi^{2}$ of 61.7 for 51 d.o.f. with $k T_{\mathrm{e}}=4.6 \mathrm{keV}$, while the normalization of the COMPTT component increases to $3.53 \times 10^{-2}$, and $N_{\mathrm{H}}^{\mathrm{CTT}}$ increases to $3.6 \times 10^{23}$ atom $\mathrm{cm}^{-2}$. However, the fact that in only 1 out of 6 trials (three spectra with 2 models) the $\chi^{2}$ is reduced with a significance of $6 \%$ probably means that there is no compelling reason to treat the temperature of 
Table 3. Results of fitting the simple absorption model to the dip spectra. $r_{\text {in }}(\cos i)^{0.5}$ assumes a distance of $15 \mathrm{kpc}$.

\begin{tabular}{llll}
\hline Parameter & Deep & Medium & Shallow \\
\hline$N_{\mathrm{H}}^{\mathrm{CTT}}\left(10^{22}\right.$ atom $\left.\mathrm{cm}^{-2}\right)$ & $30.8 \pm 4.7$ & $23.1 \pm_{3.2}^{3.6}$ & $33.6 \pm_{6.5}^{7.8}$ \\
$I_{\mathrm{CTT}}\left(10^{-2}\right)$ & $2.12 \pm_{0.14}^{0.19}$ & $3.15 \pm 0.23$ & $3.49 \pm_{0.42}^{0.52}$ \\
$N_{\mathrm{H}}^{\mathrm{DBB}}\left(10^{22}\right.$ atom cm $\left.{ }^{-2}\right)$ & $<0.07$ & $<0.16$ & $0.07 \pm 0.04$ \\
$r_{\text {in }}(\cos i)^{0.5}$ & $0.77 \pm 0.03$ & $2.21 \pm_{0.05}^{0.03}$ & $3.02 \pm 0.03$ \\
Flux $(2-10 \mathrm{keV})\left(\mathrm{erg} \mathrm{cm}^{-2} \mathrm{~s}^{-1}\right)$ & $0.95 \times 10^{-10}$ & $3.3 \times 10^{-10}$ & $5.0 \times 10^{-10}$ \\
$\chi^{2} /$ d.o.f. & $66.1 / 52$ & $107.2 / 69$ & $74.8 / 77$ \\
\hline
\end{tabular}

either the COMPTT or disk-blackbody components as free parameters during dips.

The uncertainty in the value of $r_{\text {in }}(\cos i)^{0.5}$ obtained from the fits to the dip spectra (see Table 3 ) is much smaller than that obtained from the fits to the persistent emission spectrum (Table 2), although there are far fewer counts in the dip spectra. This results from fixing the two temperatures in the model which is used to fit the dip spectra, while the temperatures are free parameters in the fit to the persistent emission spectrum. The high value for $r_{\text {in }} \cos i$ obtained from the shallow dip spectrum (compared to that obtained from the quiescent spectrum), is hard to understand physically. However, there is a strong correlation between $T_{\text {in }}$ and $r_{\text {in }} \cos i$ and fixing $T_{\text {in }}$ to 1.76 (best-fit value $+90 \%$ confidence uncertainty) results in a value for $r_{\text {in }} \cos i$ which is within the uncertainty quoted in Table 2.

We note that the $\chi^{2}$ for the medium dip spectrum is formally unacceptable with a $0.2 \%$ probability that the model describes the data. This might be caused by the large amount of spectral variability during the intervals over which the medium dip spectrum was selected (see Fig. 1). This could result in the "mixing" of spectra with different amounts of absorption and normalizations, which cannot be well fit by the chosen model.

Inspection of the fit residuals for the deep-dip spectrum reveals clear structure around $6.4 \mathrm{keV}$, indicative of the presence of an Fe-K line. The addition of this line reduces the $\chi^{2}$ from 66.1 for 52 d.o.f. to 47.1 for 49 d.o.f., which is significant at $>99 \%$ confidence. The equivalent width of the line is $330 \pm 155 \mathrm{eV}$, the number of photons in the line is $4.4 \times 10^{-4} \mathrm{~cm}^{-2} \mathrm{~s}^{-1}$, the line energy is $6.44 \pm 0.18 \mathrm{keV}$, and its width $(\sigma)$ is $310 \pm{ }_{310}^{270} \mathrm{eV}$. The only parameter of the continuum model which changes significantly due to the addition of the line is the normalization of the COMPTT component, which decreases by $14 \%$. There is no evidence for lines at $6.44 \mathrm{keV}$ in the other dip spectra with $1 \sigma$ upper limits to the number of line photons of $5.0 \times 10^{-5} \mathrm{~cm}^{-2} \mathrm{~s}^{-1}$ and $7.1 \times 10^{-5} \mathrm{~cm}^{-2} \mathrm{~s}^{-1}$ for the medium and shallow dip intervals, respectively.

\subsubsection{Complex absorption model}

Since fitting the simplest model that provides statistically acceptable fits to the X 1658-298 dip spectra gives results which are inconsistent with the physical interpretation of
Table 4. Fit results of the complex absorption model to the dip spectra. Units are the same as in Table 3.

\begin{tabular}{llll}
\hline Parameter & Deep & Medium & Shallow \\
\hline$N_{\mathrm{H}}^{\mathrm{DBB}}$ & $37.3 \pm 2.5$ & $17.8 \pm 2.3$ & $<0.09$ \\
$N_{\mathrm{H}}^{\mathrm{CTT}}$ & $123 \pm{ }_{20}^{13}$ & $>148$ & $13.1 \pm{ }_{2.2}^{3.4}$ \\
$f$ & $0.91 \pm 0.01$ & $0.31 \pm 0.02$ & $0.47 \pm 0.04$ \\
$\chi^{2} /$ d.o.f. & $70.6 / 53$ & $115.5 / 70$ & $75.7 / 79$ \\
\hline
\end{tabular}

the complex continuum model of Church \& BałucińskaChurch (1995), where the softer component is rapidly and strongly absorbed, we also fit this model to the same dip spectra. Since the blackbody and cutoff power-law model does not provide a good description of the persistent emission spectrum (see Table 2), we instead used the best-fit COMPTT and disk-blackbody model for this comparison. If the physical situation is similar, with the disk-blackbody originating from a compact region and the Comptonized component from an extended region, this should allow a meaningful comparison to be made with the complex continuum model.

In this case the model flux is given by:

$\mathrm{e}^{-\sigma N_{\mathrm{H}}}\left(I_{\mathrm{DBB}} \mathrm{e}^{-\sigma N_{\mathrm{H}}^{\mathrm{DBB}}}+I_{\mathrm{CTT}}\left(f \mathrm{e}^{-\sigma N_{\mathrm{H}}^{\mathrm{CTT}}}+(1-f)\right)\right)$,

where $I_{\mathrm{DBB}}$ and $I_{\mathrm{CTT}}$ are the normalizations of the disk-blackbody and COMPTT components, $f$ the covering fraction of the COMPTT component, and $\sigma$ the photo-electric absorption cross-sections of Morrison \& McCammon (1983). Note that the fundamental differences with the simple absorption model that is used in Sect. 5.2.1 are that the COMPTT component is partially absorbed and $I_{\mathrm{DBB}}$ and $I_{\mathrm{CTT}}$ are fixed at the values measured during the persistent emission.

The best-fit values of $\chi^{2}$ are slightly worse than the fit results from the simple model, but show similar trends, e.g., the medium dip spectrum is not well modeled (probably because of the same reason as discussed in Sect. 5.2.1). The absorbing column for the disk-blackbody component smoothly increases from $<9 \times 10^{20}$ atom $\mathrm{cm}^{-2}$ during the shallow dip to $(3.73 \pm 2.5) \times 10^{23}{\text { atom } \mathrm{cm}^{-2}}^{-2}$ during the deep dip. The trends in the absorption column and covering fraction of the COMPTT model are not as clear with the absorbing column increasing from $\left(13.1 \pm{ }_{2.2}^{3.4}\right) \times 10^{22}$ atom $\mathrm{cm}^{-2}$ during the shallow dip to 


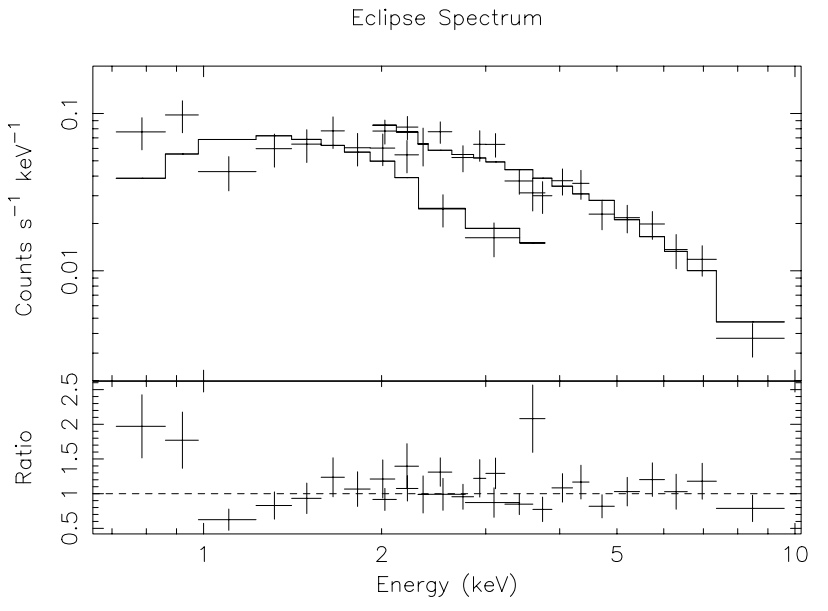

Fig. 8. The LECS and MECS eclipse spectrum of X 1658-298 together with the best-fit absorbed power-law model.

$>10^{23}$ atom $\mathrm{cm}^{-2}$ elsewhere. The covering fraction ranged from $0.31 \pm 0.02$ during the medium dip to $0.91 \pm 0.01$ during the deep dip. The results indicate that at all times the COMPTT component is obscured by a significantly higher column than the disk-blackbody. This confirms the same result obtained using the simple absorption model (see Sect. 5.2.1) and is in contrast to the results previously obtained by applying the complex-continuum model to the dip spectra of other dipping sources.

\subsection{Eclipse spectrum}

The spectral shape of the emission during eclipse was investigated. Data from all four observed eclipses were combined to provide exposure times of 1.7 and $2.3 \mathrm{ks}$ for the LECS and MECS, respectively. The HPGSPC and PDS spectra do not contain significant signals and were excluded from this analysis. The resulting spectrum can be fit by an absorbed $\left(N_{\mathrm{H}}=(3.4 \pm 1.6) \times 10^{21}\right.$ atom $\left.\mathrm{cm}^{-2}\right)$ power-law with $\alpha=2.0 \pm{ }_{0.1}^{0.2}$ (Fig. 8). The $\chi^{2}$ is 35.3 for 25 d.o.f. and the $2-10 \mathrm{keV}$ luminosity is $4.3 \times 10^{35} \mathrm{erg} \mathrm{s}^{-1}$ for a distance of $15 \mathrm{kpc}$. This is $2.7 \pm 1.0 \%$ of the persistent value. Note the structured residuals in the lowest energy channels of the LECS. This may suggest the presence of e.g., a dust scattered component, but the low count rate does not allow us to reliably investigate this further. We note that similar low-energy residual structure may also be present in the deep dip spectrum (cf. Figs. 6 and 8). The $90 \%$ confidence upper limit to the number of photons in a $6.44 \mathrm{keV}$ emission line is $7.6 \times 10^{-5} \mathrm{~cm}^{-2} \mathrm{~s}^{-1}$.

\section{X-ray bursts}

Nine X-ray bursts are visible in the light curve (see Fig. 1). The first burst is the brightest with a peak flux measured during $2 \mathrm{~s}$ intervals of 1.5 times that of the other bursts. This burst was studied in detail to see if there was evidence for radius expansion which can be used to estimate the distance to the source. Light curves were produced in different energy bands. These show that some struc- ture is present in the burst, especially at higher energies (which is usually indicative of radius expansion). Timesliced spectra (with $2 \mathrm{~s}$ time bins) of the burst where then obtained and the resulting spectra fit (after subtracting the persistent emission obtained in a $500 \mathrm{~s}$ interval before the burst as background) with a blackbody model. However, the limited statistics do not allow us to clearly ascertain whether this burst shows radius expansion. The blackbody radius during the brightest $2 \mathrm{~s}$ of the burst is numerically larger than the other values, but is consistent (at the $1 \sigma$ level) with being constant. Using a flux estimate obtained from the two seconds when the burst is brightest $\left(1.4 \times 10^{-8} \mathrm{erg} \mathrm{cm}^{-2} \mathrm{~s}^{-1}\right)$ and assuming that this corresponds to the theoretical Eddington limit for a $1.4 M_{\odot}$ neutron star with a hydrogen atmosphere emitting isotropically a distance of $11 \mathrm{kpc}$ is obtained. Using the empirically observed Eddington luminosity $\left(3.0 \times 10^{38} \mathrm{erg} \mathrm{s}^{-1}\right)$ of a sample of bursters for which the distance is known (Lewin et al. 1993), a distance of $13 \mathrm{kpc}$ is obtained.

These estimates agree well with the distance of $15 \mathrm{kpc}$ obtained by Cominsky (1981) who assumed that the 13 burst sources observed by SAS-3 were uniformly distributed around the galactic center. The distances to individual burst sources, relative to the galactic center (assumed to be at $9 \mathrm{kpc}$ ), were obtained by comparing individual properties with those of the sample. We have searched the literature for reports of other radius expansion bursts from X 1658-298; none were found. We conclude that the luminosity of the first burst is likely to be at, or close to, the Eddington limit. Note that our estimates can be interpreted as upper limits to the distance, if the burst is not a radius expansion burst (and was thus emitting at below the Eddington luminosity).

\section{Discussion}

We present results of a 2000 August BeppoSAX observation of X 1658-298. The persistent emission can best be modeled by the summation of disk-blackbody and

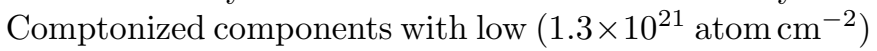
absorption. The best-fit to a 2-20 keV RXTE Proportional Counter Array spectrum obtained 16 months earlier is to a cutoff power-law model with $\alpha=2.1 \pm 0.1, N_{\mathrm{H}}=$ $(5.0 \pm 0.6) \times 10^{22}$ atom $\mathrm{cm}^{-2}$ and $E_{\mathrm{c}}=8.6 \pm 0.6 \mathrm{keV}$ (WSB). When the same model is fit to the BeppoSAX NFI

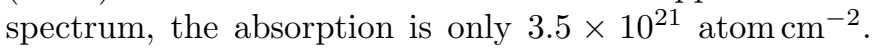
A straightforward interpretation of this striking difference is that during the early stages of the outburst the $\mathrm{X}$-ray emission is seen through a strongly thickened accretion disk, while by the time of the BeppoSAX observation the disk had thinned and the central emission regions are directly viewed during non-dipping intervals. The observed 2-20 keV luminosity during the RXTE observation of $2.8 \times 10^{37} \mathrm{erg} \mathrm{s}^{-1}$ (WSB, converted from their quoted flux, which we assume to be the absorbed value) is indeed $\sim 40 \%$ higher than our 2-20 keV luminosity, consistent 
with a higher mass-accretion rate during the earlier phase of the outburst.

The spectrum of the persistent emission from X 1658298 can be described by disk-blackbody and Comptonized components (the COMPTT model). However the $\chi^{2}$ is not formally acceptable and low-level structure in the residuals is present which is probably due to the inadequate modeling of the source spectrum. The addition of Gaussian line features improves the fit (without constraining the parameters of these features very well). This implies the presence of additional complexity in the spectrum of X 1658-298. The combination of a disk-blackbody or blackbody model and a Comptonized component has been fit a large number of LMXRB spectra (see e.g., Guainazzi et al. 1998; in 't Zand et al. 1999b; Barret et al. 2000; Sidoli et al. 2001), although a physical interpretation of the disk-blackbody parameters is not straightforward (see e.g., Merloni et al. 2000). It is possible that the deviations from the smooth continuum in X 1658-298 are caused by intervening material, through which the emission regions are observed. The reasons why this intervening material is prominent in this source is probably due to the high inclination angle - the line of sight passes close to the surface of the accretion disk. Higher quality data from Chandra or XMM-Newton are needed to shed further light on the nature of these features.

The spectra during dips can be reasonably well approximated with the persistent model, with only the COMPTT component undergoing strong additional absorption of $\sim 3 \times 10^{23}$ atom $\mathrm{cm}^{-2}$. The normalizations of both spectral components decreases as the dipping gets deeper. The normalization of the disk-blackbody component shows the strongest dependence on total flux, decreasing to $\sim 5 \%$ of the persistent value during the deep dip, compared to $\sim 60 \%$ for the COMPTT component. This would suggest that the disk-blackbody component originates from a more compact region, while part of the Comptonized region is still observed directly during dips. In this case it is likely that during the deepest dipping intervals the diskblackbody is no longer viewed directly, the $5 \%$ of emisssion remaining could be X-rays scattered into our line of sight in an extended scattering region (the same region responsible for the COMPTT component perhaps) in a similar way to the $2.7 \%$ of emission still visible during eclipse. The compact nature of the disk-blackbody and the extended nature of the Comptonizing region agrees with results obtained by e.g., Church et al. (1997) and Church \& Bałucińska-Church (1995) with the "progressive covering" model. However, the strong additional absorption suffered by the COMPTT component is in contrast to the results of this model, where the (disk-)blackbody component rapidly undergoes strong additional absorption during dips.

This additional COMPTT absorption is however consistent with the picture emerging from XMM-Newton observations of the LMXRB EXO 0748-676, where the Comptonized component undergoes significant extra absorption during dipping intervals (Bonnet-Bidaud et al. 2001), while the softer thermal component does not.
However, during the EXO 0748-676 eclipses the thermal component remains, implying that it is the extended component in this system. It is possible to extend this picture to X 1658-298 to obtain a consistent picture of all the spectral changes observed during the dips. We first assume that the disk-blackbody component is actually an extended "thermal" component similar to that seen by Bonnet-Bidaud et al. (2001) from EXO 0748-676 and arranged in a thin layer above and below the accretion disk. In order that the normalization of the thermal component reduces to $\sim 5 \%$ of its persistent emission value during deep dips, $\sim 95 \%$ of the projected emission area should be obscured by the dense outer regions of the accretion disk. Assuming that the disk can be regarded as a dense solid body, then only a small fraction of the thermal flux will undergo absorption due to skin effects. This could explain why no additional absorption is detected from this component. Simulations of the structure of X-ray irradiated disks reveal that such sharp transitions in vertical structure may arise due to an ionization instability (Done \& Nayakshin 2001).

The Comptonized component does, however, suffer from additional absorption and from a $\sim 40 \%$ reduction in normalization during deep dips. Assuming that this emission originates from a spherical region centered on the neutron star, then during deep dips $\sim 40 \%$ of the emission region should be obscured by the solid accretion disk as with the thermal component. The remaining $\sim 60 \%$ is located away from the line of sight to the accretion disk. It is likely that the material in the line of sight to the Comptonized component is highly turbulent due to the interaction of the gas stream from the companion star with the outer accretion disk and the ionization instability described by Krolik et al. (1981). This results in the formation of small relatively cool clouds embedded in a hotter medium (Frank et al. 1987). The passage of these clouds in front of the Comptonized region could result in the observed strong increase in absorption.

While this model is totally ad-hoc, it is an attempt to explain the complexity observed during the dips. It does imply a particular geometry for the emitting regions, and in particular for the thermal component that must be located close to the accretion disk so that it can be almost totally obscured. We note that the two systems where this model may be applicable are the two eclipsing systems which are seen at inclinations angles of $\sim 75-80^{\circ}$, compared to the pure dip sources which are viewed at inclinations angles of $\sim 60-75^{\circ}$ (e.g., Frank et al. 1997). We speculate that the different viewing geometry may be one reason for the different spectral behavior during dips of X 1658-298 and EXO 0748-676 and the other dip sources.

An interesting feature is the appearance of a strong Fe-K line during the X 1658-298 deep dip, whose energy is consistent with that of neutral or moderately ionized iron. Its origin (and absence during the other dip spectra) is rather difficult to understand, but maybe related to the amount of intervening material in the line of sight where 
substantial reprocessing of the continuum radiation can take place.

The BeppoSAX ephemeris does not require a $\dot{P}$ term, in contrast to the ephemeris of WSB. This is probably due to the extremely uneven sampling (and possibly a small underestimate of the uncertainties) of the arrival times in WSB, resulting in an artificial solution. We note that small (random?) deviations from a linear ephemeris are probably present, but this depends sensitively on the assumed uncertainties in the arrival times. The observed deviations may be similar in nature to those observed from EXO 0748-676 (see Hertz et al. 1997).

Acknowledgements. The BeppoSAX satellite is a joint Italian and Dutch programme. L. Sidoli acknowledges an ESA Research Fellowship. We thank L. Cominsky for discussions and the anonymous referee for helpful suggestions.

\section{References}

Augusteijn, T., Freyhammer, L., \& in 't Zand, J. 1999, IAU Circ., 7138

Barret, D., Olive, J. F., Boiron, L., et al. 2000, ApJ, 553, 329

Boella, G., Chiappetti, L., Conti, G., et al. 1997, A\&AS, 122, 327

Bonnet-Bidaud, J. M., Haberl, F., Ferrando, F., Bennie, P. J., \& Kendziorra, E. 2001, A\&A, 365, L282

Church, M. J., \& Bałucińska-Church, M. 1995, A\&A, 300, 441

Church, M. J., Dotani, T., Bałucińska-Church, M., et al. 1997, ApJ, 491, 388

Church, M. J., Parmar, A. N., Bałucińska-Church, M., et al. 1999, A\&A, 338, 556

Church, M. J. Proc. Cospar 2000, Adv. Space Research [astro-ph/0012411]

Church, M. J., \& Bałucińska-Church, M. 2001, A\&A, 369, 915

Cominsky, L. R. 1981, Ph.D. Thesis, MIT

Cominsky, L. R., \& Wood, K. S. 1984, ApJ, 283, 765

Cominsky, L. R., \& Wood, K. S. 1989, ApJ, 337, 485
Cottam, J., Kahn, S. M., Brinkman, A. C., den Herder, J. W., \& Erd, C. 2000, A\&A, 365, L277

Dickey, J. M., \& Lockman, F. J. 1990, ARA\&A, 28, 215

Done, C., \& Nayakshin, S. 2001, ApJ, 546, 419

Doxsey, R., Grindlay, J., Griffiths, R., et al. 1979, ApJ, 228, $\mathrm{L} 67$

Frank, H., King, A. R., \& Lasota, J.-P. 1987, A\&A, 178, 127

Frontera, F., Costa, E., Dal Fiume, D., et al. 1997, A\&AS, 122, 371

Guainazzi, M., Parmar, A. N., Oosterbroek, T., et al. 1998, A\&A, 339, 802

Hertz, P., Wood, K. S., \& Cominsky, L. R. 1997, ApJ, 486, 1000

In 't Zand, J., Heise, J., Smith, M. J. S., et al. 1999a, IAU Circ., 7138

In 't Zand, J. J. M., Verbunt, F., Strohmayer, T. E., et al. 1999b, A\&A, 345, 100

Krolik, J., McKee, C. F., \& Tarter, C. B. 1981, ApJ, 249, 422

Lewin, W. H. G., Hoffmann, J. A., \& Doty, J. 1976, IAU Circ., 2994, 2

Lewin, W. H. G. 1979, in Advances in Space Exploration, X-ray Astronomy, ed. W. A. Baity, \& L. E. Peterson (Oxford: Pergamon), vol. 3, 133

Lewin, W. H. G., van Paradijs, J., \& Taam, R. E. 1993, Space Sci. Rev., 62, 223

Mitsuda, K., Inoue, H., Koyama, K., et al. 1984, PASJ, 36, 741

Manzo, G., Guarrusso, S., Santangelo, A., et al. 1997, A\&AS, 122,341

Merloni, A., Fabian, A. C., \& Ross, R. R. 2000, MNRAS, 313, 193

Morrison, D., \& McCammon, D. 1983, ApJ, 270, 119

Parmar, A. N., White, N. E., Giommi, P., \& Gottwald, M. 1986, ApJ, 308, 199

Parmar, A. N., Martin, D. D. E., Bavdaz, M., et al. 1997, A\&AS, 122, 309

Sidoli, L., Parmar, A. N., Oosterbroek, T., et al. 2001, A\&A, 368, 451

Titarchuk, L. 1994, ApJ, 434, 570

Valinia, A., \& Marshall, F. E. 1998, ApJ, 505, 134

Wachter, S., Smale, A. P., \& Bailyn, C. 2000, ApJ, 534, 367

Wijnands, R., Strohmayer, T., \& Franco, L. M. 2001, ApJ, 549, L71 